\title{
Effect of Freezing Rate on Physical Stability of Lyophilized Cationic Liposomes
}

\author{
Yukio Aso* and Sumie YoshiokA \\ National Institute of Health Sciences; 1-8-1 Kamiyoga, Setagaya, Tokyo 158-8501, Japan.
}

Received October 1, 2004; accepted December 8, 2004; published online December 16, 2004

Factors affecting the storage stability of lyophilized cationic liposomes were investigated using liposomes prepared with various excipients and by different freezing rates, either quick freezing (freezing by immersion into liquid nitrogen) or slow freezing (cooling to $-50^{\circ} \mathrm{C}$ at a rate of $-10^{\circ} \mathrm{C} / \mathrm{h}$ ). Increases in the particle size of cationic liposomes observed during freeze-drying were inhibited by the addition of sucrose, trehalose and sucrose-dextran mixtures $(1: 1$ and $2: 1$ by weight). The storage instability of the formulations, as indicated by changes in particle size, was affected by their glass transition temperature $\left(T_{\mathrm{g}}\right)$. Addition of high- $T_{\mathrm{g}}$ excipients resulted in smaller increases in the particle size, indicating improvement of storage stability. The storage stability of cationic liposome formulations was also affected by freezing rate. Formulations prepared by slow freezing exhibited better stability. Longer shear relaxation times were observed for formulations prepared by slow freezing compared with those prepared by quick freezing. This indicates that formulations prepared by slow freezing have a lower matrix mobility, which may result in better storage stability. $T_{\mathrm{g}}$ or ${ }^{1} \mathrm{H}-\mathrm{NMR}$ relaxation measurements could not detect differences in matrix mobility between formulations prepared by different freezing rates. Shear relaxation measurements seem to be a useful method for evaluating the storage stability of cationic liposome formulations.

Key words cationic liposome; stability; lyophilization; shear relaxation time; molecular mobility

Cationic lipid has attracted much attention as a non-viral DNA vector. Cationic liposome-DNA complex (lipoplex), however, is usually unstable in solution and forms aggregates during long-term storage at room temperature, resulting in the loss of its transfection ability. ${ }^{1,2)}$ Stability of lipoplex during freeze-drying and subsequent storage can be improved by lyophilization with lyoprotectants such as sucrose. ${ }^{3-5)}$ The stabilizing effect of these compounds on liposomes during lyophilization has been attributed to incorporation of liposomes into glass matrices and hydrogen bonding between the excipient and the polar head group of lipids. ${ }^{6}$ ) Therefore, the storage stability of lyophilized cationic liposomes is expected to be improved by addition of excipients with higher glass transition temperature $\left(T_{\mathrm{g}}\right)$, which yield glass matrices with a lower molecular mobility.

It is also known that process parameters such as freezing rate affect the stability of lyophilized formulations. It has been reported that the amount of drug entrapped in lyophilized liposomes is higher for formulations prepared by slow freezing than for those prepared by quick freezing. ${ }^{7)}$ The effect of freezing rate depended on the lipid composition and was most pronounced for rigid liposomes. The storage stability of lyophilized tissue-type plasminogen activator was also affected by freezing rate. ${ }^{8}$ ) Faster freezing caused a larger surface area of the freeze-dried cake, and the storage stability of the incorporated protein was proportional to the surface area of the freeze-dried cake.

This paper describes the effects of excipients with different $T_{\mathrm{g}}$ as well as the effect of freezing rate on the storage stability of lyophilized cationic liposomes. Changes in the particle size of cationic liposomes were determined as a measure of storage instability. ${ }^{1} \mathrm{H}-\mathrm{NMR}$ relaxation time and shear relaxation time of the freeze-dried cakes were also determined. The storage stability of cationic liposomes is discussed in terms of the molecular mobility and visco-elastic property (matrix mobility) of the freeze-dried cakes.

\section{Experimental}

Materials $N$-[1-(2,3-Dioleoyloxy) propyl]- $N, N, N$-trimethyl-ammonium chloride (DOTAP), cholesterol, sucrose, trehalose and dextran were purchased from Sigma (St. Louis, MO, U.S.A.).

Preparation of Cationic Liposomes ${ }^{3)}$ DOTAP $(250 \mathrm{mg})$ and cholesterol $(125 \mathrm{mg})$ were dissolved in chloroform (about $5 \mathrm{ml}$ ). The chloroform was evaporated with a stream of nitrogen gas to make a thin film of the lipids. The film was further dried under vacuum for $1 \mathrm{~h}$ and hydrated in $50 \mathrm{ml}$ of water. The lipid suspension was incubated at $50{ }^{\circ} \mathrm{C}$ for $10 \mathrm{~min}$ and extruded through polycarbonate membranes (Millipore, Billerica, MA, U.S.A.) with pore sizes of 0.6 and $0.2 \mu \mathrm{m}$, which were connected in series.

Preparation of Lyophilized Liposome Formulations Sucrose, trehalose and sucrose-dextran mixtures $(1: 1$ or $2: 1$ by weight) were used as excipients for lyophilized liposome formulations. Equal volumes of excipient solution $(10 \mathrm{w} / \mathrm{w} \%)$ and liposome suspension were mixed. Aliquots $(500 \mu \mathrm{l})$ of the mixture were frozen in polypropylene tubes by immersion into liquid nitrogen (quick freezing) or by cooling on the shelf of a freeze drier (Freezevac C-3; Tozai Tsusho, Tokyo) at a rate of $-10^{\circ} \mathrm{C} / \mathrm{h}$ to $-50{ }^{\circ} \mathrm{C}$ (slow freezing). Frozen samples were dried under a vacuum of approximately $5 \mathrm{~Pa}$. The shelf temperature was maintained at $-40{ }^{\circ} \mathrm{C}$ for $24 \mathrm{~h}$, $-20^{\circ} \mathrm{C}$ for $16 \mathrm{~h}, 0^{\circ} \mathrm{C}$ for $6 \mathrm{~h}$, and then $20^{\circ} \mathrm{C}$ for $6 \mathrm{~h}$.

Stability Study of Lyophilized Cationic Liposomes The water content of the lyophilized formulations was adjusted by storage at $25^{\circ} \mathrm{C}$ and $23 \%$ RH for $1 \mathrm{~d}$. The samples were stored at 40 or $25^{\circ} \mathrm{C}$ in a thermostatic chamber and withdrawn at appropriate intervals. The storage stability of liposomes was evaluated from changes in particle size. Particle size was determined at $25^{\circ} \mathrm{C}$ by dynamic light scattering with a DLS-7000 system (Otsuka Electronics Co. Ltd., Osaka). The viscosity of the rehydrated liposome suspension, required for the calculation of particle size, was determined with a model AR-1000 rheometer (TA Instruments, Inc., New Castle, DE, U.S.A.). The $T_{\mathrm{g}}$ values of the formulations are summarized in Table 1.

Physicochemical Properties of Lyophilized Liposome Formulations. Determination of the Complex Shear Modulus The visco-elastic property (matrix mobility) of the freeze-dried cakes was examined with the model AR-1000 rheometer. A freeze-dried cake was placed on the sample platform of the instrument and compressed to about $440 \mu \mathrm{m}$ thickness with a flat plate geometry $(40 \mathrm{~mm}$ in diameter). An oscillating stress of $40 \mathrm{~Pa}$ was applied to the sample over a frequency range of 0.01 to $600 \mathrm{radian} / \mathrm{s}$. The effect of sample size, which was smaller than the plate size of the geometry used, on the determination of shear relaxation time was considered to be negligible, since shear relaxation time was estimated from relative changes in the shear modulus determined as a function of stress frequency.

${ }^{1}$ H-NMR Relaxation Measurements In order to determine the molecu- 
lar mobility of the freeze-dried cakes, spin-lattice relaxation times in laboratory frame $\left(T_{1}\right)$ and rotating frame $\left(T_{1 \rho}\right)$ were measured at $27^{\circ} \mathrm{C}$ with a model JNM-MU25 spectrometer (JEOL DATUM, Tokyo) operated at a ${ }^{1} \mathrm{H}$ resonance frequency of $25 \mathrm{MHz}$. A freeze-dried cake was placed in an NMR tube $\left(10 \mathrm{~mm}\right.$ outer diameter) and dried at $25^{\circ} \mathrm{C}$ for $18 \mathrm{~h}$ under vacuum before measurement. $T_{1}$ was measured by the inversion-recovery method. A spin locking field of $1 \mathrm{mT}$ was applied to the samples for $T_{1 \rho}$ measurement.

DSC Analysis The $T_{\mathrm{g}}$ of freeze-dried cakes was determined with a model 2920 differential scanning calorimeter (TA Instruments, Inc.) equipped with a refrigerator cooling accessory. Temperature and heat flow calibration of the instrument was performed with indium. Approximately $3 \mathrm{mg}$ of freeze-dried cake was weighed into a hermetic sample pan, which was stored at $25^{\circ} \mathrm{C}$ and $23 \% \mathrm{RH}$ for $24 \mathrm{~h}$ and then sealed. Samples were heated at a rate of $20^{\circ} \mathrm{C} / \mathrm{min}$ from -30 to $200^{\circ} \mathrm{C}$. The DSC cell was purged with nitrogen gas at $30 \mathrm{ml} / \mathrm{min}$ during measurement.

Water Vapor Sorption Measurements Time profiles of water vapor sorption for lyophilized liposome formulations containing sucrose and trehalose were measured at $25^{\circ} \mathrm{C}$ and $10 \% \mathrm{RH}$ with a GM-300 gravimetric sorption analyzer (VTI Corp., Hialeah, FL, U.S.A.). Approximately $25 \mathrm{mg}$ of freeze-dried cake was placed in a sample holder and dried at $25^{\circ} \mathrm{C}$ under vacuum. When no change in the weight of the cake was observed over $5 \mathrm{~min}$, the cake was considered to be in a dry state. The cake was exposed to water vapor equivalent to $10 \% \mathrm{RH}$. The weight of the cake was measured at intervals of $30 \mathrm{~s}$.

\section{Results and Discussion}

Effect of Excipients on Stability of Lyophilized Cationic Liposomes Figure 1 shows the effect of freeze-thaw and freeze-drying on the particle size of cationic liposomes. No significant difference in particle size was observed after the freeze-thaw cycle, regardless of the presence of excipients. Increases in the particle size, however, were observed after rehydration of liposomes lyophilized without excipient. On the other hand, liposomes lyophilized with excipients exhibited similar particle sizes before and after freeze-drying, indicating that all the excipients studied stabilized cationic liposomes against aggregation during drying.

Figure 2 shows the storage stability of cationic liposomes lyophilized with the excipients. Increases in particle size were observed during storage at $40^{\circ} \mathrm{C}$ except for the trehalose formulation prepared by slow freezing. The liposome

Table 1. $T_{\mathrm{g}}$ of Lyophilized Cationic Liposome Formulations ${ }^{a)}$

\begin{tabular}{lcc}
\hline \hline \multirow{2}{*}{ Excipient } & \multicolumn{2}{c}{$T_{\mathrm{g}}\left({ }^{\circ} \mathrm{C}\right)^{b)}$} \\
\cline { 2 - 3 } & Slow freezing & Quick freezing \\
\hline Sucrose & 32.3 & 31.9 \\
Trehalose & 48.4 & 49.0 \\
Suc-Dex $(2: 1)$ & 43.5 & 43.7 \\
Suc-Dex $(1: 1)$ & 59.7 & 59.9 \\
\end{tabular}

a) Water content was adjusted by storage at $25^{\circ} \mathrm{C}$ and $23 \% \mathrm{RH}$ for $1 \mathrm{~d}$. b) $T_{\mathrm{g}}$ values reported were average of two determinations. formulations containing sucrose, the $T_{\mathrm{g}}$ of which $\left(32^{\circ} \mathrm{C}\right)$ was lower than the storage temperature, were least stable. The formulations containing trehalose, the $T_{\mathrm{g}}$ of which $\left(49^{\circ} \mathrm{C}\right)$ was higher than the storage temperature, were more stable than the sucrose formulations. Addition of dextran to sucrose resulted in a higher $T_{\mathrm{g}}\left(43^{\circ} \mathrm{C}\right.$ for $2: 1$ sucrose-dextran mixture and $60^{\circ} \mathrm{C}$ for $1: 1$ mixture) and increased the stability of the liposome formulations. These results indicate that the stability of cationic liposome formulations is closely correlated with the mobility of the formulation matrix as indicated by $T_{\mathrm{g}}$. The formulation containing trehalose prepared by quick freezing exhibited increases in the particle size, but no significant increase was observed for the trehalose formulation prepared by slow freezing, indicating that the storage stability of lyophilized cationic liposomes is affected by the freezing rate.

Figure 3 shows a photograph of lyophilized cationic liposomes containing sucrose or trehalose stored at $25^{\circ} \mathrm{C}$ and $23 \% \mathrm{RH}$ for 1 year. Shrinkage of the freeze-dried cake was observed for sucrose formulations. The extent of shrinkage was larger for the sample prepared by quick freezing. No significant shrinkage of freeze-dried cakes was observed for trehalose formulations and sucrose-dextran formulations (data not shown), which had $T_{\mathrm{g}}$ values higher than those of sucrose formulations. Figure 4 shows the particle size of liposomes after storage at $25^{\circ} \mathrm{C}$ and $23 \% \mathrm{RH}$ for 1 year. The trehalose and sucrose-dextran formulations were stable, but an increase in the particle size was observed for sucrose formulations during storage at $25^{\circ} \mathrm{C}$ (approximately $8^{\circ} \mathrm{C}$ lower than the $T_{\mathrm{g}}$ of the sucrose formulation). These results indicate that the sucrose formulations have a sufficient degree of matrix mobility to cause liposome aggregation even at $25^{\circ} \mathrm{C}$, a temperature lower than the $T_{\mathrm{g}}$ of the formulation.

\section{Molecular Mobility and Matrix Mobility of Cationic}

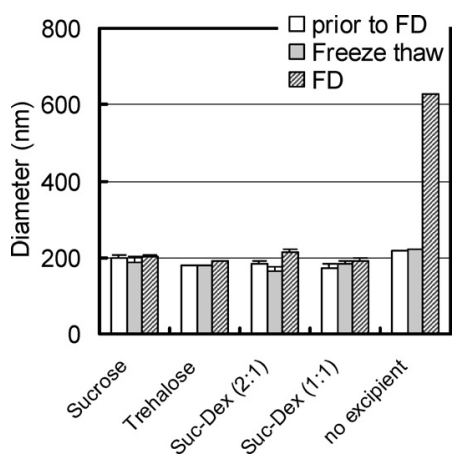

Fig. 1. Effect of Freeze-Thaw and Freeze-Drying on the Particle Size of Cationic Liposomes

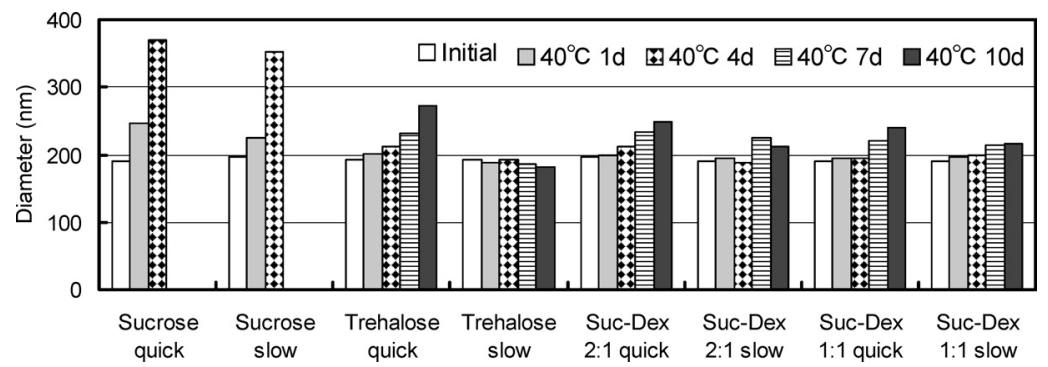

Fig. 2. Effect of Storage at $40^{\circ} \mathrm{C}$ on the Particle Size of Cationic Liposomes 
Liposome Formulations The storage stability of lyophilized cationic liposomes was affected by freezing rate, such that formulations prepared by quick cooling were less stable than those prepared by slow cooling, as shown in Figs. 2 -4. To gain an insight into the mechanism of the effect of freezing rate on the stability of cationic liposome formulations, the mobility of the formulation matrices was examined by ${ }^{1} \mathrm{H}-\mathrm{NMR}$ relaxation and shear relaxation measurements. Table 2 shows the $T_{1}$ and $T_{1 \rho}$ of cationic liposome formulations. $T_{1}$ and $T_{1 \rho}$ are measures of molecular mobility on time

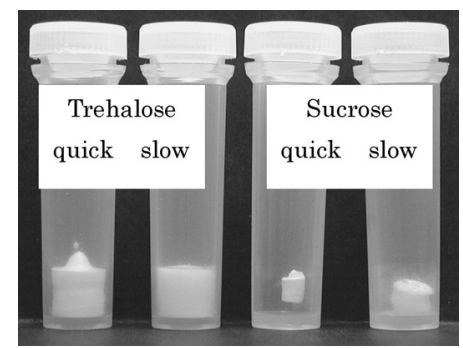

Fig. 3. Appearance of Freeze-Dried Cakes of Cationic Liposomes Containing Sucrose or Trehalose after Storage at $25^{\circ} \mathrm{C}$ and $23 \% \mathrm{RH}$ for 1 Year

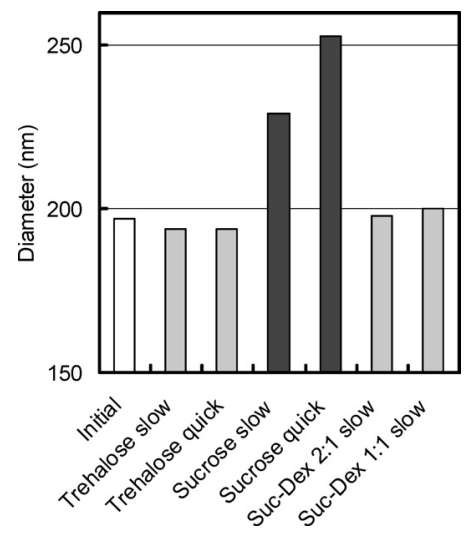

Fig. 4. Effect of Storage at $25^{\circ} \mathrm{C}$ and $23 \%$ RH for 1 Year on Particle Size of Cationic Liposomes

Table 2. ${ }^{1} \mathrm{H}$-NMR Relaxation Time of Cationic Liposome Formulations

\begin{tabular}{clcc}
\hline \hline Excipient & & $T_{1}(\mathrm{~s})$ & $T_{1 \rho}(\mathrm{ms})$ \\
\hline \multirow{2}{*}{ Sucrose } & Slow & 0.29 & 4.0 \\
& Quick & 0.28 & 4.0 \\
Trehalose & Slow & 0.28 & 4.4 \\
& Quick & 0.30 & 4.6 \\
\hline
\end{tabular}

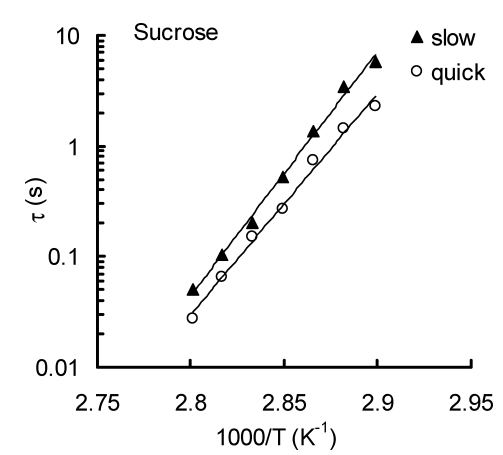

scales of the order of $\mathrm{MHz}$ and mid $\mathrm{kHz}$, respectively. ${ }^{9)} T_{1}$ and $T_{1 \rho}$ of the formulations did not change with freezing rate within experimental error, indicating that freezing rate has little effect on the molecular mobility reflected in $T_{1}$ and $T_{1}$.

The mobility of formulation matrices on longer time scales than those reflected in NMR spin-lattice relaxation times was determined by measuring the frequency dependence of the shear modulus of lyophilized cationic liposomes (the visco-elastic property of the formulations). The frequency-dependent shear modulus $\left(G^{*}(\omega)\right)$ of amorphous indomethacin has been shown to characterize the time scales of molecular motion of indomethacin in the amorphous state. ${ }^{10)}$ The storage modulus $\left(G^{\prime}\right.$, a real part of $\left.G^{*}(\omega)\right)$ of visco-elastic materials increases with increasing frequency of shear stress, and the loss modulus $\left(G^{\prime \prime}\right.$, an imaginary part of $\left.G^{*}(\omega)\right)$ exhibits a maximum. Visco-elastic materials behave as a viscous fluid and a rigid solid against low and high frequency stress, respectively. The shear relaxation time, the reciprocal of maximum frequency (in radian/s) of $G^{\prime \prime}$, can be used as a measure of mobility. Figure 5 shows the typical frequency dependence of the shear modulus of the cationic liposome formulation containing sucrose measured at $74^{\circ} \mathrm{C}$ in the dry state. Figure 6 shows the temperature dependence of shear relaxation time for the cationic liposome formulations containing sucrose and trehalose measured under dry conditions. The relaxation time of the formulation prepared by slow freezing was longer than that of the formulation prepared by quick freezing. The effect of freezing rate on the shear relaxation time was also observed in the presence of moisture. Figure 7 shows the frequency dependence of $G^{\prime \prime}$ for the cationic liposome formulation containing sucrose stored at $25^{\circ} \mathrm{C}$ and $23 \% \mathrm{RH}$ for $1 \mathrm{~d}$. The formulation prepared by

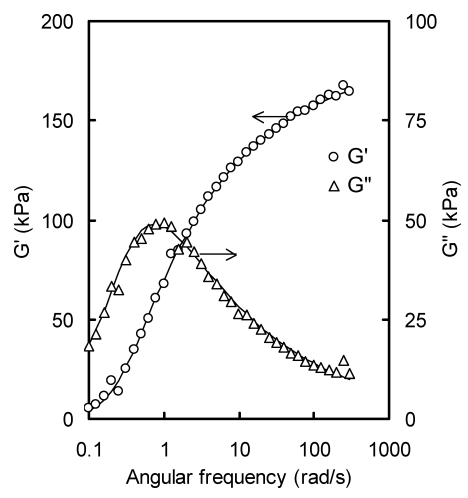

Fig. 5. Frequency Dependence of the Shear Modulus of a Cationic Liposome Formulation Containing Sucrose Measured at $74{ }^{\circ} \mathrm{C}$ in the Dry State

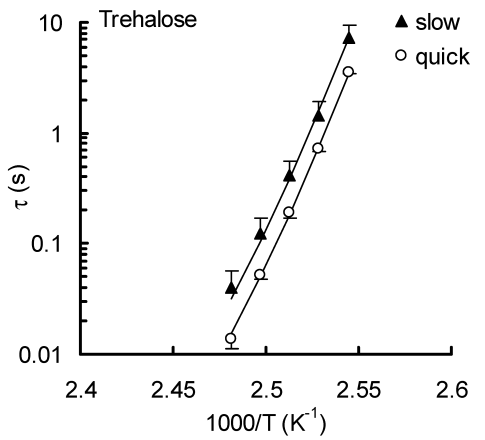

Fig. 6. Shear Relaxation Time of Cationic Liposome Formulations Measured under Dry Conditions 


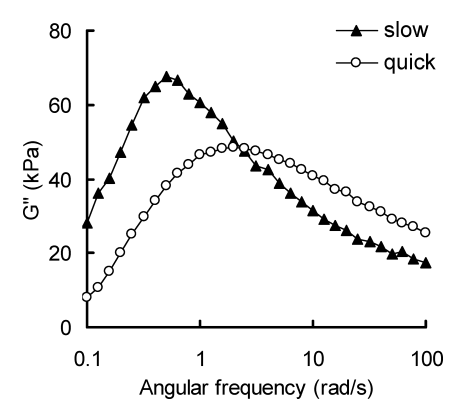

Fig. 7. Frequency Dependence of the Loss Modulus $\left(G^{\prime \prime}\right)$ of a Cationic Liposome Formulation Containing Sucrose at $30^{\circ} \mathrm{C}$

The water content was adjusted by storage at $25^{\circ} \mathrm{C}$ and $23 \% \mathrm{RH}$ for $1 \mathrm{~d}$.

quick freezing exhibited a maximum at 2 radian/s, whereas the formulation prepared by slow freezing exhibited a maximum at $0.4 \mathrm{radian} / \mathrm{s}$. These results indicate that formulation matrices prepared by slow freezing have a lower mobility than those prepared by quick freezing. Temperature dependence of $G^{\prime \prime}$ supports this speculation. $G^{\prime \prime}$ at a frequency of $0.1 \mathrm{radian} / \mathrm{s}$ showed a maximum at $21^{\circ} \mathrm{C}$ for the sucrose formulation prepared by quick freezing, whereas $26^{\circ} \mathrm{C}$ for the sucrose formulation prepared by slow freezing. The maximum temperature corresponds to the temperature at which relaxation time of $10 \mathrm{~s}$ (inverse of $0.1 \mathrm{radian} / \mathrm{s}$ ) is observed. Therefore, the relaxation time at $25^{\circ} \mathrm{C}$ is considered to be longer than $10 \mathrm{~s}$ for the sucrose formulation prepared by slow freezing. In contrast, shorter relaxation time than $10 \mathrm{~s}$ is expected for the sucrose formulation prepared by quick freezing. This difference in matrix mobility resulting from different freezing rates may cause a difference in storage stability. The difference in matrix mobility of cationic liposome formulations prepared by different freezing rates could be detected by shear relaxation time, but not by $T_{\mathrm{g}}$ and ${ }^{1} \mathrm{H}-\mathrm{NMR}$ relaxation measurements (Tables 1,2 ). This finding suggests that the aggregation rate of lyophilized liposomes may correlate more closely with matrix mobility as measured by the shear relaxation time than with molecular mobility as measured by the spin-lattice relaxation time.

Figure 8 shows the time course of water vapor sorption for the cationic liposome formulations containing sucrose and trehalose stored at $25^{\circ} \mathrm{C}$ and $10 \% \mathrm{RH}$. The water content at equilibrium was not affected by freezing rate. The weight of the formulations prepared by quick freezing, however, reached a plateau within $40 \mathrm{~min}$, whereas it took more than $300 \mathrm{~min}$ to reach equilibrium for formulations prepared by slow freezing. The difference in water sorption rate indicates that the ratio of surface area to volume for the formulations prepared by quick freezing is larger than that for the formulations prepared by slow freezing. Similar differences in the surface area of freeze-dried cakes have been reported for lyophilized tissue-type plasminogen activator formulations. ${ }^{8)}$ Such dependence of the surface area on freezing rate may cause the different mobility of the formulation matrices, as indicated by the different shear relaxation times. Formulations with lager surface area may be more susceptive to changes in the $T_{\mathrm{g}}$ upon local temperature fluctuations and/or local humidity fluctuations. Differences in the susceptibility may affect the shrinkages of freeze-dried cakes and the sta-
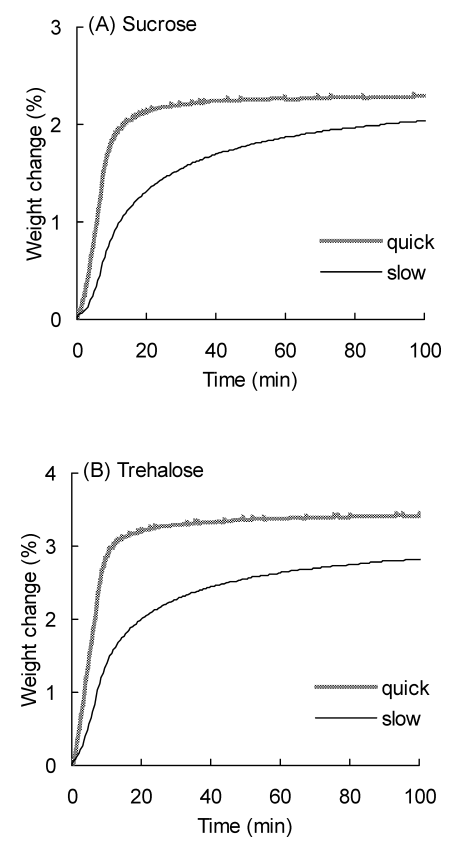

Fig. 8. Time Courses of Water Vapor Sorption of Cationic Liposome Formulations Stored at $25^{\circ} \mathrm{C}$ and $10 \% \mathrm{RH}$

bility of liposomes during storage.

In conclusion, the storage instability of lyophilized cationic liposomes, as indicated by change in particle size, was affected by the $T_{\mathrm{g}}$ of the formulations. Formulations containing high- $T_{\mathrm{g}}$ excipients exhibited better storage stability. The storage stability of lyophilized cationic liposomes was also affected by freezing rate. Longer shear relaxation times were observed for formulations prepared by slow freezing compared with those prepared by quick freezing, indicating that formulations prepared by slow freezing have a lower matrix mobility. The lower matrix mobility of the formulations prepared by slow freezing may result in better storage stability. $T_{\mathrm{g}}$ or ${ }^{1} \mathrm{H}-\mathrm{NMR}$ relaxation measurements could not detect these differences in matrix mobility. Shear relaxation measurements appear to be useful for evaluating the storage stability of cationic liposome formulations.

Acknowledgements A part of this work was supported by a Grant-inaid for Research on Health Sciences Focusing on Drug Innovation from The Japan Health Sciences Foundation.

\section{References}

1) Anchordoquy T. J., Koe G. S., J. Pharm. Sci., 89, 289-296 (2000).

2) Lai E., van Zanten J. H., J. Pharm. Sci., 91, 1225-1232 (2002).

3) Li B., Li S., Tan Y., Stolz D. B., Watkins S. C., Block L. H., Huang L., J. Pharm. Sci., 89, 355-364 (2000).

4) Anchordoquy T. J., Carpenter J. F., Kroll D. J., Arch. Biochem. Biophys., 348, 199-206 (1997).

5) Molina M. C., Allison S. D., Anchordoquy T. J., J. Pharm. Sci., 90, 1455-1455 (2001).

6) Crowe J. H., Leslie S. B., Crowe L. M., Cryobiology, 32, 355-366 (1994).

7) van Winden E. C., Zhang W., Crommelin D. J., Pharm. Res., 14, 1151-1160 (1997).

8) Hsu C. C., Nguyen H. M., Yeung D. A., Brooks D. A., Koe G. S., Bewley T. A., Pearman R., Pharm. Res., 12, 69-77 (1995).

9) Horii F., "Solid State NMR of Polymers," Chap. 3, ed. by Ando I., Elsevier, Amsterdam, 1998, pp. 51-81.

10) Andronis V., Zografi G., Pharm. Res., 14, 410-414 (1997). 